\title{
Economic feedback model predictive control of wave energy converters
}

\author{
Siyuan Zhan, Guang Li, Member, IEEE and Colin Bailey
}

\begin{abstract}
This paper proposes an economic feedback model predictive control (MPC) scheme to improve energy conversion efficiency of wave energy converters (WECs) and guarantee their safe operation over a wide range of sea conditions. The proposed MPC control law consists of two terms: one state feedback gain designed offline to maximize operating range and one term calculated online to maximize the energy output. Compared with the existing MPC strategies developed for the WEC control problem, the proposed feedback economic MPC strategy has the following distinguishing advantages: (i) The satisfaction of safety constraints and the recursive feasibility can be guaranteed to ensure WEC's safe operation in a large range of sea states. (ii) The novel MPC can notably improve energy production efficiency. (iii) The controller design procedure is more convenient and straightforward compared with the existing MPC strategies. The efficacy of the proposed MPC strategy is demonstrated by numerical simulations with a point absorber as a case study. By comparison with a representative existing MPC strategy, the proposed economic MPC can significantly improve energy output.
\end{abstract}

Index Terms-Wave energy, model predictive control, energy maximization

\section{INTRODUCTION}

Wave energy is a promising renewable energy source in many countries, with over $2 \mathrm{TW}$ coastal power potential estimated in the world [1]. To meet the increasing demand of clean energy worldwide, tremendous research efforts have been focused on generating electricity from waves. Although many different types of wave energy converters (WECs) have been invented, wave energy is still not mature for commercialization compared with other renewable energy sources, e.g. wind energy and solar energy [2]. Two of the main challenges of wave energy technology are the low energy conversion efficiency in particular sea states and limited safe operational sea states due to the high risk of device damage. [3].

It is well accepted that efficient and reliable control strategies for WECs are crucial to improve their energy conversion efficiency and safety in a range of sea states. Early wave energy control methods are mainly based on impedance matching principle, that is, the maximal energy output can be achieved when the natural frequency of a WEC matches the dominant frequency of the incoming waves. However, these WEC control methods are most effective for idealized

S. Zhan, G. Li and C. Bailey are with the Department of Engineering and Material Science, Queen Mary University of London, London, E1 4NS, UK. Address all correspondence to Guang Li (g.li@qmul.ac.uk).

This work was supported by the Newton Advanced Fellowship from The Royal Society, UK, under Grant NA160436 and a contract with Wave Energy Scotland on control system design of wave energy converters. regular waves and can become complicated and inconvenient to implement in real sea conditions.

The WEC control problem is essentially a noncausal energy maximization (EM) control problem subject to the limits of the motion of the device and the capacity of the actuator and it can be resolved by the recently advanced optimal control strategies [4], [5] to approximate the noncausal optimality identified in [6]. By noncausal we mean the current optimal control input is dependent on the future wave profile [6]. The EM control problem for WECs can be potentially resolved by economic model predictive control (MPC) [7]. Originally motivated by process control problems [8], the topic of economic MPC received extensive research attentions with remarkable theoretical developments achieved [9]-[11]. Inspired by the idea of economic MPC, some WEC MPC methods [12], [13] have been developed to tackle the WEC control problem that can directly maximize energy output. However, most of these existing WEC MPC strategies are open-loop MPC methods, which have two major drawbacks restricting their applications. Firstly, they cannot guarantee recursive feasibility. Briefly speaking, the feasibility of an MPC controller is determined by the current state of the WEC, the current and predicted wave profile, and the constraints on state and input. When the MPC for WEC control is not feasible, no solution can be found by the MPC optimisation algorithm, and constraints have to be violated to find a feasible solution. In real applications, significant constraint violations can cause catastrophic failure for WEC operations. The existing MPC strategies for WEC control cannot guarantee recursive feasibility, which means the feasible solution of the MPC algorithms cannot be ensured to be found at each sampling instant. Secondly, the range of sea states that the WEC can safely operate in is significantly influenced by the selection of objective functions. In fact, maximizing energy output and maximizing the range of safe operational sea states are conflicting control objectives. This is because, to maximize the energy output, large oscillations of the WECs are more desirable, but also more likely to bring infeasibility and violation of safety constraints problems. The existing open-loop MPC strategies with one objective function combining both objectives require a suitable trade-off of the two objectives, which is not only difficult to determine, but also result in both limited operational range and reduced energy conversion efficiency.

In this paper, we propose an economic feedback MPC framework to resolve the aforementioned drawbacks of the existing open-loop MPC. Researches [14]-[16] show that to guarantee the recursive feasibility and robust satisfaction of safety constraints for systems subject to persistent disturbance, 
the MPC has to be implemented over state feedback policies, instead of open-loop actions. Based on this concept, the proposed economic feedback MPC scheme explicitly preserve recursive feasibility and satisfaction of constraints for safety concerns. The economic feedback MPC law consists of two terms: a state feedback gain that is designed offline and one term calculated by online optimization. The novelties of the proposed method include:

1) The recursive feasibility and constraints satisfaction are guaranteed all the time for the given sea states.

2) The mutually conflicting control objectives can be separately treated, which makes the controller design and tuning more convenient and straightforward. To maximize the range of safe operational sea states is accomplished by design of a state feedback gain and the energy output is maximized through resolving the online optimization problem.

The above features enable the proposed feedback economic MPC to fully utilize the potential of a WEC and the capacity of power take-off (PTO) and enhance the survivability, so that the unit cost of the generated electricity can be significantly reduced. Moreover, the proposed economic feedback MPC design method also simplifies the optimal tuning procedure of the WEC controller, and the tuning weights can be computed via offline optimization conveniently. In comparison, a manually tuned standard MPC controller can only be based on trial-anderror and cannot achieve the desired trade-off. Only a welltuned existing standard MPC cannot guarantee recursive feasibility and constraint satisfaction, which is clearly demonstrated in the simulation.

The rest of the paper is organized as follows. In Section II, the dynamic modeling of a single WEC is established and the corresponding constrained optimal control problem is formulated. The economic feedback MPC scheme is formulated in Section III and the implementation is demonstrated in Section IV. Numerical simulations are demonstrated in Section V to verify the efficacy of the proposed methods. Finally, the paper is concluded in Section VI.

Notations: We denote the set of integers from $m$ to $n$ by $\mathbb{I}_{[m, n]}$; the set of non-negative integers by $\mathbb{I}_{\geq 0}$; the set of all real numbers by $\mathbb{R}$; the set of all non-negative real numbers by $\mathbb{R}_{>0}$. The space of real $n$-dimensional vectors is denoted by $\mathbb{R}^{n}$. Let $[a, b]$ denote the column vector $\left[a^{T}, b^{T}\right]^{T},[A, B]$ denote matrix $\left[A^{T}, B^{T}\right]^{T}$, and $a_{i \mid k}$ denote elements at time $i$ predicted/estimated at time $k$. Let $\boldsymbol{a}_{k}$ denote a column vector $\left[a_{k \mid k}, \ldots, a_{k+n_{p}-1 \mid k}\right]$, where $n_{p}$ is the wave prediction step.

For subsets $\mathbb{A} \subset \mathbb{R}^{n}$ and $\mathbb{B} \subset \mathbb{R}^{n}$, the Minkowski set addition is defined by $\mathbb{A}+\mathbb{B} \triangleq\{a+b: a \in \mathbb{A}, b \in \mathbb{B}\}$. The $\mathrm{P}$ subtraction is defined by $\mathbb{A} \sim \mathbb{B} \triangleq\left\{a \in \mathbb{R}^{n}: a+b \in \mathbb{A}, \forall b \in\right.$ $\mathbb{B}\}$. For $\mathbb{A} \subset \mathbb{R}^{n}$ and matrix $M$ of compatible dimensions, $M \mathbb{A}:=\{M a: a \in \mathbb{A}\}$.

\section{WEC DYNAMICS MODELING AND PROBLEM SETUP}

We present the economic MPC method using a point absorber (PA) as a case study, which is constrained in heave motion only for harnessing wave energy. Note that the economic feedback MPC method proposed in this paper is generic

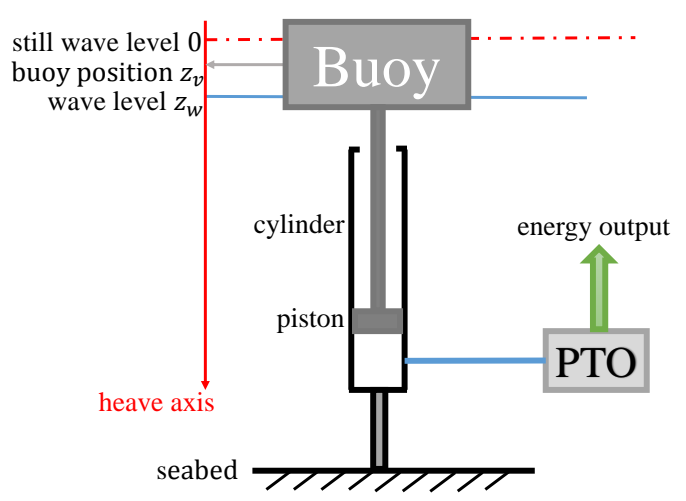

Fig. 1. Schematic diagram of a point absorber

and can be applied to other types of WECs. We briefly explain the working principle of the PA, whose schematic diagram is shown in Fig. 1. The heave displacement of the buoy and the wave surface level are denoted as $z_{v}$ and $z_{w}$ respectively.

The buoy is floating on the sea surface, which is attached to a piston within a hydraulic cylinder fixed to the sea bed. The persistent wave excitation force drives the buoy, which creates a relative heave motion between the piston and the cylinder. The kinetic energy is captured by a PTO mechanism. The PTO mechanisms used to capture WEC energy vary for different WEC designs, while the most popular ones are based on direct linear generators [17], [18] and hydraulic motors connected to electricity generators [19], [20]. Since the generator torque or force is proportional to the force acting on the piston $F_{u}$, we use $F_{u}$ as the control input without loss of generality. We do not consider nonlinear hydrodynamics in this paper to simplify the analysis and controller design. The linearized model has sufficient fidelity for small and medium sea states and some well-designed WECs, e.g. M4 [21].

The WEC modeling method used in this paper is similar to [22] and [23]. The free-body diagram of the float is shown in Fig. 2. By applying Newton's second law, we have

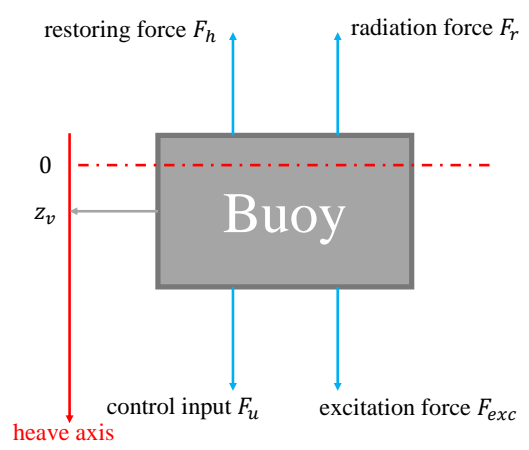

Fig. 2. Free body dynamic diagram of the float

$$
m_{s} \ddot{z}_{v}=-F_{h}-F_{r}+F_{e x c}+F_{u}
$$

where $m_{s}$ is the float mass; the restoring force $F_{h}$ is computed by

$$
F_{h}=k_{s} z_{v}
$$


with the hydrostatic stiffness $k_{s}=\rho g S$. Here $\rho$ is water density, $g$ is gravitational acceleration, and $S$ is the crosssectional area of the float. $F_{r}$ and $F_{e x c}$ are the frequencydependent radiation force and excitation force respectively. The control oriented modeling of the radiation force $F_{r}$ is determined by

$$
F_{r}:=m_{\infty} \ddot{z}_{v}+F_{d}
$$

where $m_{\infty}$ is the added mass at infinite frequency; $F_{d}$ represents the convolutional term of the radiation force, which can be computed by

$$
F_{d}:=\int_{-\infty}^{t} h_{r}(\tau) \dot{z}_{v}(t-\tau) d \tau
$$

$h_{r}$ is radiation impulse response that can be computed via hydraulic software packages, such as NEMOH [24]. The convolutional term in (4) $F_{d}$ can be approximated by a causal finite dimensional state-space model

$$
\begin{aligned}
& \dot{x}_{r}=A_{r} x_{r}+B_{r} \dot{z}_{v} \\
& F_{d}=C_{r} x_{r} \approx \int_{-\infty}^{t} h_{r}(\tau) \dot{z}_{v}(t-\tau) d \tau
\end{aligned}
$$

where $\left(A_{r}, B_{r} C_{r}, 0\right)$ and $x_{r} \in \mathbb{R}^{n_{r}}$ are the state-space realization and the state respectively.

With the state-space modeling of (5), the control oriented full-order state-space model of (1) can be represented by

$$
\left\{\begin{array}{l}
\dot{x}=A_{c} x+B_{u c} u+B_{w c} w \\
v=C_{v} x \\
z=C_{z} x
\end{array}\right.
$$

where $w:=F_{e x c}, z:=z_{v}, v:=\dot{z}_{v}, x:=\left[z_{v}, \dot{z}_{v}, x_{r}\right]$ and

$$
\begin{aligned}
A_{c} & =\left[\begin{array}{ccc}
0 & 1 & 0 \\
-\frac{k_{s}}{m} & 0 & -\frac{C_{r}}{m} \\
0 & B_{r} & A_{r}
\end{array}\right] \quad B_{w c}=B_{u c}=\left[\begin{array}{c}
0 \\
\frac{1}{m} \\
0
\end{array}\right] \\
C_{z} & =\left[\begin{array}{lll}
0 & 1 & 0_{1 \times n_{r}}
\end{array}\right]
\end{aligned}
$$

with $m:=m_{s}+m_{\infty}$.

To formulate the MPC scheme, the continuous time model (6) needs to be converted to a discrete-time model with a sampling time $t_{s}$

$$
\left\{\begin{array}{l}
x_{k+1}=A x_{k}+B_{u} u_{k}+B_{w} w_{k} \\
z_{k}=C_{z} x_{k} \\
v_{k}=C_{v} x_{k}
\end{array}\right.
$$

Here $z_{k}$ represents the heave displacement and $v_{k}$ represents the heave velocity. The WEC linear optimal control development in the remaining paper will be based on this discrete time model.

With the WEC dynamic modeling direction defined in Fig. 2 , the power output at time instant $k$ is

$$
P_{k}:=-v_{k} u_{k}
$$

and energy output for a period from time instant 0 to $k$ can be represented as

$$
E_{k}:=-t_{s} \sum_{i=0}^{k} v_{i} u_{i}
$$

For safe operation purposes, we restrict the float's heave motion and heave velocity so that the state constraints can be expressed as

$$
\left|z_{k}\right| \leq \Phi_{\max }, \quad\left|v_{k}\right| \leq v_{\max }
$$

where $\Phi_{\max }$ and $v_{\max }$ are the float heave motion and heave velocity limits respectively. Due to the limitation of PTO mechanism capacities, the WEC is also subject to control input constraint expressed as

$$
|u| \leq u_{\max }
$$

where $u_{\max }$ is the maximal control input force acting on the piston.

In summary, the WEC controller design objective is to maximize the energy output expressed by (10) subject to constraints (11) for sea conditions with maximum heave elevation profiles satisfying

$$
|w| \leq w_{\max }
$$

with a $n_{p}$-step wave excitation force prediction

$$
\boldsymbol{w}_{k}:=\left[w_{k \mid k}, w_{k+1 \mid k}, \ldots, w_{k+n_{p}-1 \mid k}\right] .
$$

Here $w_{\max }$ represents the maximum of the wave heave magnitude that allows the safe operation of the WEC. A larger $w_{\max }$ means the WEC can be safely working in higher sea states. This WEC noncausal optimal control problem can be tackled by recursively solving the following constrained optimal control problem based on MPC's receding horizon concept:

$$
\begin{array}{ll}
\boldsymbol{u}_{k}^{*}= & \arg \min _{\left[u_{k \mid k} \ldots, u_{k+n_{p}-1 \mid k}\right]} \sum_{i=0}^{n_{p}-1} v_{k+i \mid k} u_{k+i \mid k} \\
\text { s.t. } \quad & \quad 8) \quad\left|z_{k+i}\right| \leq \Phi_{\max },\left|v_{k+i}\right| \leq v_{\max },\left|u_{k+i}\right| \leq u_{\max } \\
& \forall\left|w_{k+i}\right| \leq w_{\max } \forall i \in \mathbb{I}_{\left[0, n_{p}-1\right]}
\end{array}
$$

Here, the first element of $\boldsymbol{u}_{k}^{*}$ is used as the control input at time instant $k$.

Note that the MPC problem for WECs is substantially different from the conventional MPC problem, which invalidates the direct employment of the existing MPC algorithms. This is mainly due to the following distinguishing features of the WEC MPC control problem:

1) Whilst the traditional MPCs usually aim to regulate states or track a reference leading to a convex quadratic programming $(\mathrm{QP})$, the control objective of WEC MPC is to maximize the energy output, whose stage cost $v_{k+i \mid k} u_{k+i \mid k}$ may not be necessarily definite leading to a non-convex cost function.

2) In a WEC MPC design problem, due to the presence of persistent wave excitation force, the asymptotic convergence to a particular optimal operation state cannot be easily achieved. To ensure safe operation of a WEC, the constraints on states and control input (11) are required to be satisfied for all possible incoming waves in a particular sea condition satisfying (12). 
3) The WEC MPC resolves a non-causal optimal control problem online. The information of short-term wave prediction $\boldsymbol{w}_{k}$ can be incorporated into the WEC controller to further improve the control performance.

4) Unlike the traditional MPC, where the effects of external disturbances need to be attenuated, the WEC MPC aims to absorb energy from the incoming waves as the excitation disturbances by achieving resonance for energy maximization.

\section{FEEdBACK ECONOMIC MPC STRUCTURE DESIGN}

As discussed in Section I, the existing WEC MPC [12], [13], generally cannot guarantee the recursive feasibility, and the state feedback MPC policy can solve this issue. However, in a WEC control problem, due to the unavailability of direct measurements of all the states, especially those corresponding to the radiation dynamics $D_{r}(s)$. A state observer is required to retrieve the full state information. Considering the aforementioned aspects, we propose the economic feedback MPC framework

$$
u_{k}=F \hat{x}_{k}+c_{k}
$$

where $F$ is the offline designed feedback coefficients; $\hat{x}$ is the estimated state; $c$ is the auxiliary variable to be online calculated.

Remark 1. The economic feedback MPC framework allows us to separately maximize the WEC control system's ability to cope with harsh sea conditions to cover a wide operational range of sea states via the design of feedback coefficient $F$ and maximize the potential energy output subject to constraints by online resolving an optimization problem to derive $c_{k}$.

\section{A. State observer design}

To estimate the full information of the states, based on the availability of wave prediction (13), a Luenberger observer is designed with the following form

$$
\hat{x}_{k+1}=A \hat{x}_{k}+B_{u} u_{k}+B_{w} w_{k \mid k}+L\left(y_{k}-C \hat{x}_{k}\right),
$$

where $w_{k \mid k}$ is the estimation of current wave excitation and defined in (13); $y_{k}$ is the measured output

$$
y_{k}=C x_{k}
$$

with $C:=\left[C_{z}, C_{v}\right]$, where $C_{z}$ and $C_{w}$ defined in (6). Here $(A, C)$ is assumed to be observable. $L$ is the observer gain designed with the radius $\rho(A-L C)<1$, so that the information of the real state $x_{k}$ can be recovered by estimated state $\hat{x}_{k}$ with an acceptable error $\xi_{k}:=x_{k}-\hat{x}_{k}$.

Remark 2. Note that due to the presence of input and state constraints, the separation principle of controller and observer design is no longer valid in a straightforward manner. Thus, the effects of estimation need to be explicitly studied in the controller design to avoid constraints violation.

Comparing (8) with (17), we have

$$
\xi_{k+1}=(A-L C) \xi_{k}+B_{w}\left(w_{k}-w_{k \mid k}\right)
$$

Remark 3. Since $\rho(A-L C)<1$, there exists a bound $\mathbb{E}$ such that for $\xi_{k} \in \mathbb{E}$ for all $k \geq 0$ [25], [26]. Moreover, the error $\xi_{k} \rightarrow 0$ with $k \rightarrow 0$ and $w_{k \mid k}=w_{k}$.

Assumption 1. We assume that the the observer gain $L$ is properly designed so that $\mathbb{E}$ is sufficiently small.

This assumption holds throughout the paper and can be easily satisfied since $(A, C)$ is observable and the poles of the observer can be located at desirable locations by $L$. Based on the observer design theory, a large gain causes fast convergence of the state estimation error. However, the implementation of an observer with a large gain can be very demanding for the computational speed of a microprocessor. Thus, a good trade-off for this gain needs to be found for real-time implementation.

\section{B. Constraints handling}

Although a numerically tractable MPC algorithm can be designed based on (14) with the preview information of incoming waves, e.g. [13], the recursive feasibility for optimization problem (14) cannot be guaranteed. This drawback significantly increases the risk of catastrophic device damage, which reduces the confidence of wave energy control engineers to implement this type of MPC algorithm in real applications.

To proceed with the constraint handling design, the state and input constraints (11) and the disturbance bound (12) are represented by $x \in \mathbb{X}, u \in \mathbb{U}$ and $w \in \mathbb{W}$ respectively, where $\mathbb{X}, \mathbb{U}$ and $\mathbb{W}$ are defined by

$$
\begin{aligned}
\mathbb{X} & :=\left\{x \in \mathbb{R}^{n_{x}}:\left|C_{z} x_{k}\right| \leq \Phi_{\max } \text { and }\left|C_{v} x_{k}\right| \leq v_{\max }\right\} \\
\mathbb{U} & :=\left\{u \in \mathbb{R}:\left|u_{k}\right| \leq u_{\max }\right\} \\
\mathbb{W}: & :=\left\{w \in \mathbb{R}:\left|w_{k}\right| \leq w_{\max }\right\}
\end{aligned}
$$

Here $C_{z}$ and $C_{w}$ are defined in (6); $\Phi_{\max }$ and $v_{\max }$ are defined in (11); $w_{\max }$ are defined in (12).

To guarantee the satisfaction of constraints defined by (11) and the recursive feasibility, we further impose the constraint satisfaction (14c) for all $i \geq 0$.

Lemma 1. The requirement of $x_{k+i} \in \mathbb{X}, u_{k+i} \in \mathbb{U}$ for all $i \geq 0$ and for all possible incoming wave profiles in certain sea conditions $w_{k+i} \in \mathbb{W}$ can be guaranteed by imposing tightened constraints $\bar{x}_{k+i \mid k} \in \mathbb{X}_{k}, \bar{u}_{k+i \mid k} \in \mathbb{U}_{i}$ for $i \in \mathbb{I}_{\left[0, n_{p}-1\right]}$ and $\bar{x}_{n_{p}+k \mid k} \in \mathbb{X}_{T}$ on the auxiliary state and input trajectories predicted using the following auxiliary system

$$
\begin{aligned}
\bar{x}_{k+i+1 \mid k} & =A \bar{x}_{k+i \mid k}+B_{u} \bar{u}_{k+i \mid k} \\
\bar{u}_{k+i \mid k} & =F \bar{x}_{k+i \mid k}+c_{k+i \mid k} \\
\bar{x}_{k \mid k} & =\hat{x}_{k}
\end{aligned}
$$

where $\hat{x}_{k}$ is the estimated state information provided by the Luenberger observer (16) and the tightened constraints are defined by

$$
\begin{array}{ll}
\mathbb{X}_{i}:=\mathbb{X} \sim \mathbb{E} \sim \mathbb{D}_{i}, & \mathbb{U}_{i}:=\mathbb{U} \sim F \mathbb{D}_{i} \\
\mathbb{D}_{i}:=\sum_{j=0}^{i-1} A_{F}^{j}\left[B_{w} \mathbb{W}+\left(A_{L}-A_{F}\right) \mathbb{E}\right], & \mathbb{X}_{T}:=\Sigma \sim \mathbb{D}_{n_{p}}
\end{array}
$$


where $A_{F}:=\left(A+B_{u} F\right), A_{L}:=(A-L C) ; \mathbb{X}, \mathbb{U}$ and $\mathbb{W}$ are defined in (19); $\mathbb{E}$ is defined in Remark $3 ; \Sigma$ is is the maximal output admissible set (MOAS) defined by

$$
\Sigma:=\left\{\begin{array}{ll} 
& x_{k+1}=A_{F} x_{k}+\eta_{k}, \\
x_{0} \in \mathbb{X}: & x_{k} \in \mathbb{X} \sim \mathbb{E}, u_{k} \in \mathbb{U}, \forall k \in \mathbb{I} \geq 0, \\
& \forall \eta_{k} \in B_{w} \mathbb{W}+\left(A_{L}-A_{F}\right) \mathbb{E}
\end{array}\right\}
$$

Proof. By comparing (20) with (8), we have

$$
\begin{aligned}
& x_{k+i}=\bar{x}_{k+i \mid k}+\xi_{k+i \mid k}+\sum_{j=1}^{i} A_{F}^{j-1} B_{w} w_{k+j} \\
& u_{k+i}=\bar{u}_{k+i \mid k}+F\left(\sum_{j=1}^{i} A_{F}^{j-1} B_{w} w_{k+j}\right)
\end{aligned}
$$

The tightened constraints (21) are satisfied for the state and input trajectories determined by (20) such that $x_{k+i}-\xi_{k+i \mid k} \in$ $\mathbb{X} \sim \mathbb{E}$ and $u_{k+i} \in \mathbb{U}$ for $i \in \mathbb{I}_{\left[0, n_{p}-1\right]}$ and $x_{k+n_{p}}-\xi_{k+n_{p} \mid k} \in$ $\Sigma$. By choosing the terminal local controller to be $u_{k+i}=$ $F x_{k+i}$ for $i \geq n_{p}$, and with the definition of (22), we have for all $i \in \mathbb{I}_{\geq 0}$,

$$
x_{k+i}-\xi_{k+i \mid k} \in \mathbb{X} \sim \mathbb{E}, \quad u_{k+i} \in \mathbb{U}
$$

Since the state estimation error is bounded by $\xi_{k+i \mid k} \in \mathbb{E}$, we have $x_{k+i} \in \mathbb{X}$ and $u_{k+i} \in \mathbb{U}$ for all $i \in \mathbb{I}_{\geq 0}$, which completes the proof.

Remark 4. If $F$ is designed so that $\rho\left(A+B_{u} F\right)<1$, the $M O A S$ (22) is a polytope and can be finitely determined using Algorithm 4.1 of [26].

\section{Design of F: maximization of the range of save operational sea states}

In Subsection III-B, we propose a constraint handling method for proposed MPC that can guarantee the safe operation of a WEC. The existence of such a control requires the determination of the MOAS. To maximize the range of sea states for the WEC to safely operate in, the feedback gain $F$ is designed such that the resulting MOAS exists for the largest possible disturbance $w_{\max }$, i.e.

$$
\begin{gathered}
\max _{F} w_{\max } \\
\text { s.t. } \exists \Sigma \text {, satisfies (22) }
\end{gathered}
$$

Note that (23) describes a complex optimization problem which does not have analytic solutions. Some numerical methods to approximate the optimal solution of (23) and the resultant MOAS $\Sigma(22)$ can be found in [27]-[29].

\section{Economic objective function handling: convexification of objective functions}

Note that with the original objective function defined in (14a), the resultant constrained optimization problem may not be convex, which can cause excessive computational complexity [13]. Moreover, the direct use of the original objective function can lead to a bang-bang control input, i.e. only the upper and lower limit of control input is used as the control input to achieve optimal control. The frequent switches between the upper and lower limit of control input causes challenges for the implementation of the control and increase the hardware maintenance cost. Moreover, recent studies [13] show that the non-convexity problem can be solved by appropriately modifying the objective function, which can significantly influence the control performance in some scenarios.

To tackle the aforementioned problems caused by nonconvexity, inspired by [13], we propose a method to convexify the objective function in an optimal way. In the following, we adopt the following modified objective function

$$
\sum_{i=0}^{n_{p}-1}\left\{\tilde{u}_{k+i \mid k} C_{v} \tilde{x}_{k+i \mid k}+r \tilde{u}_{k+i \mid k}^{2}\right\}
$$

where the predicted trajectories $\tilde{x}_{k+i \mid k}$ and $\tilde{u}_{k+i \mid k}$ are computed from an auxiliary system

$$
\begin{aligned}
\tilde{x}_{k+i+1 \mid k} & =A \tilde{x}_{k+i \mid k}+B_{u} u_{t+k \mid t}+B_{w} w_{k+i \mid k} \\
\tilde{u}_{k+i \mid k} & =F \tilde{x}_{k+i \mid k}+c_{k+i \mid k} \\
\tilde{x}_{k \mid k} & =\hat{x}_{k}
\end{aligned}
$$

which can be rewritten in a compact form as

$$
\boldsymbol{c}_{k}^{T} \mathcal{H} \boldsymbol{c}_{k}+\boldsymbol{c}_{k}^{T} \mathcal{F}+\mathcal{G}
$$

where $\boldsymbol{c}_{k}:=\left[c_{k \mid k}, \ldots, c_{k+n_{p}-1 \mid k}\right]$. Coefficients $\mathcal{H}, \mathcal{F}$ and $\mathcal{G}$ can be found after straightforward matrix manipulations, which are summarized in Appendix. Note that compared with the original cost function (14a), the second term is added to the modified stage cost (24) to make the objective function convex. To minimize the effect of this additive term on the overall performance, $r$ is designed so that the effect of the second term in the cost function is minimized. This goal can be accomplished by resolving the optimization problem

$$
\min _{r \geq 0}|r|, \quad \text { s.t. } \mathcal{H} \geq 0
$$

\section{IMPLEMENTATION AND RECURSIVE FEASIBILITY}

Based on the methods presented in Section III, the economic feedback MPC framework proposed in this paper is shown in Fig. 3. The design procedure is summarized in the Design Procedure and the implementation is demonstrated in Algorithm 1.

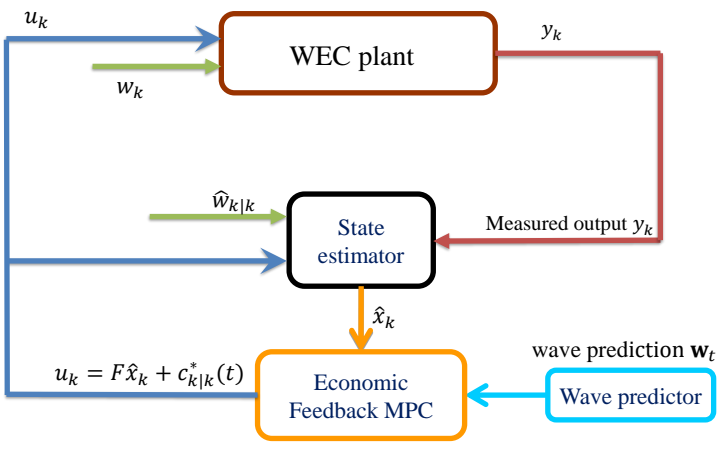

Fig. 3. WEC economic feedback MPC framework 
The existence of feasible solutions can be guaranteed by (23), which aims to find a feedback coefficient $F$ such that feasible solutions are guaranteed with a sea condition with a maximum wave excitation force $w_{\max }$.

Design Procedure: The economic feedback MPC can be designed via the following procedure.

1) Design the Luenberger observer (16) for state estimation so that the state information is recovered by the estimator (16) with a small enough estimation error $\xi_{k}$.

2) Compute the corresponding error state bound $\mathbb{E}$.

3) Design the state feedback coefficient $F$ from (23) so that the resultant control can yield feasible solutions for the largest bound of sea wave profiles $w_{\max }$. This step helps to broaden the conditions where the WEC with proposed MPC can operate.

4) Compute the MOAS $\Sigma(22)$ and the tightened state constraints $\mathbb{X}_{i}$, tightened input constraints $\mathbb{U}_{i}$ and terminal constraints $\mathbb{X}_{T}$ using (21).

5) Design the modified objective function using (27) if the original objective function (14a) is non-convex. Compute the matrices $\mathcal{H}$ and $\mathcal{F}$ (26).

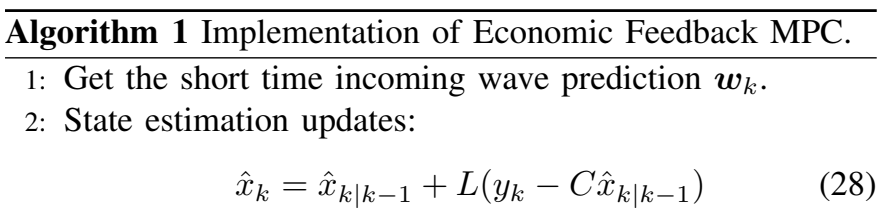

where $\hat{x}_{k \mid k-1}$ is the estimated state computed at the previous time instant $k-1$.

3: Control input updates: solve

$$
\boldsymbol{c}_{k}^{*}=\arg \min _{\boldsymbol{c}_{k}} \boldsymbol{c}_{k}^{T} \mathcal{H} \boldsymbol{c}_{k}+\boldsymbol{c}_{k}^{T} \mathcal{F}
$$$$
\text { s.t. (20) and (21) }
$$

and apply $u_{k}=F \hat{x}_{k}+c_{k \mid k}^{*}$ as the control input. Here $c_{k \mid k}^{*}$ is the first element of the optimal solution $c_{k}^{*}$.

4: State estimation update:

$$
\hat{x}_{k+1 \mid k}=A \hat{x}_{k}+B_{u} u_{k}+B_{w} w_{k \mid k}
$$

where $\hat{w}_{k}$ is the current wave measurement.

5: Go to the next time instant $k+1$ and repeat steps $1-4$.

Definition 1 (Recursive feasibility). The proposed economic feedback MPC scheme is recursively feasible, if for any $k \in$ $\mathbb{I}_{\geq 0}, \boldsymbol{c}_{k}^{*}$ solves the optimization problem (29), then there exists a feasible solution satisfying (29) at the next time instant $k+1$.

Lemma 2. The proposed economic MPC in Algorithm 1 designed following the Design Procedure is recursively feasible.

Proof. The proof of Lemma 2 can be completed if we show

$$
\boldsymbol{c}_{k+1}:=\left[c_{k+1 \mid k}^{*}, c_{k+2 \mid k}^{*}, \ldots, c_{k+n_{p}-1 \mid k}^{*}, 0\right]
$$

is a feasible solution at time instant $k+1$.

We first observe that the estimated states $\hat{x}_{k}$ and $\hat{x}_{k+1}$ satisfy

$$
\hat{x}_{k+1}+\xi_{k+1}=A_{F}\left(\hat{x}_{k}+\xi_{k}\right)+B_{u} c_{k \mid k}^{*}+B_{w} w_{k}
$$

which gives

$$
\begin{aligned}
\hat{x}_{k+1}= & A_{F} \hat{x}_{k}+B_{u} c_{k \mid k}+B_{w} w_{k}+\left(A_{F} \xi_{k}-\xi_{k+1}\right) \\
= & A_{F} \hat{x}_{k}+B_{u} c_{k \mid k}^{*}+B_{w} w_{k} \\
& +\left(A_{F} \xi_{k}-A_{L} \xi_{k}-B_{w}\left(w_{k}-w_{k \mid k}\right)\right) \\
= & A_{F} \hat{x}_{k}+B_{u} c_{k \mid k}^{*}+\eta_{k}
\end{aligned}
$$

with $\eta_{k}:=B_{w} w_{k \mid k}+\left(A_{F}-A_{L}\right) \xi_{k}$. From $x \in \mathbb{X}$ and $\xi \in \mathbb{E}$, we have $\eta_{k} \in B_{w} \mathbb{W}+\left(A_{F}-A_{L}\right) \mathbb{E}$.

With (31) and (33), we have for $i \in \mathbb{I}_{\left[1, n_{p}-1\right]}$

$$
\begin{aligned}
& \bar{x}_{k+i-1 \mid k+1}=\bar{x}_{k+i-1 \mid k}+A_{F}^{i-1} \eta_{k} \\
& \bar{u}_{k+i-1 \mid k+1}=\bar{u}_{k+i-1 \mid k}+F A_{F}^{i-1} \eta_{k}
\end{aligned}
$$

The proof can be completed following Lemma 7 from [14] with $w, F, \Phi, \mathbb{X}, \mathbb{U}$ and $\mathbb{W}$ set as $w=\eta, F=F, \Phi=$ $A_{F}, \mathbb{X}=\mathbb{X} \sim \mathbb{E}, \mathbb{U}=\mathbb{U}$ and $\mathbb{W}=B_{w} \mathbb{W}+\left(A_{F}-A_{L}\right) \mathbb{E}$, respectively.

Remark 5. In the WEC control problem, the recursive feasibility and robust constraint satisfaction are the critical features because they ensure that (i) a feasible solution of the MPC (29) always exists; (ii) the state and input constraints (11) are satisfied all the time under sea state satisfying $|w| \leq w_{\max }$.

Remark 6. The WEC MPC scheme can separately maximize the range of safe operational sea states and maximize the energy output (27) in those sea states.

\section{NUMERICAL EXAMPLES}

In this Section, three sets of numerical examples are presented to demonstrate the efficacy of the proposed MPC approach. In Subsection V-A, after the demonstration of design procedure of the proposed novel MPC, a set of simulations based on a chirping signal is presented to verify the efficacy and robustness of the proposed MPC approach. In Subsections $\mathrm{V}-\mathrm{B}$ and V-C, comparative simulations are provided between the existing WEC MPC [13] and the proposed MPC based on real sea data and Joint North Sea Wave Project (JONSWAP) spectrum [30], respectively.

The simulations in Section V are based on the model as described in (6). The parameters adopted in this simulation case are based on a widely studied benchmark WEC model firstly studied in [22] and summarized in Table I. The state space dynamic model for the frequency dependent radiation force (5) is given by

$$
\begin{aligned}
A_{r} & =\left[\begin{array}{lll}
0 & 0 & -17.9 \\
1 & 0 & -17.7 \\
0 & 1 & -4.41
\end{array}\right], \quad B_{r}=\left[\begin{array}{c}
36.5 \\
394 \\
75.1
\end{array}\right] \\
C_{r} & =\left[\begin{array}{lll}
0 & 0 & 1
\end{array}\right]
\end{aligned}
$$

A. Simulation set 1: the formulation and robustness analysis of the proposed MPC

After discretizing the system with a sampling time $t_{s}=0.1$ $\mathrm{s}$, we formulate the economic feedback MPC using Algorithm 1. A wave prediction horizon length of $t_{p}=1 \mathrm{~s}$ (equivalent to $n=10$ prediction steps) of incoming wave excitation force is 
TABLE I

THE PARAMETERS USED FOR THE WEC MODEL

\begin{tabular}{lll}
\hline Description & Notation & Values \\
\hline Stiffness & $k_{s}$ & $3866 \mathrm{~N} / \mathrm{m}$ \\
Float mass & $m_{s}$ & $242 \mathrm{~kg}$ \\
Added mass & $m_{\infty}$ & $83.5 \mathrm{~kg}$ \\
Total mass & $m$ & $325.5 \mathrm{~kg}$ \\
Control input limit & $u_{\max }$ & $5 \mathrm{kN}$ \\
Heave displacement limit & $\Phi_{\max }$ & $1 \mathrm{~m}$ \\
Heave velocity limit & $v_{\max }$ & $10 \mathrm{~m} / \mathrm{s}$ \\
\hline
\end{tabular}

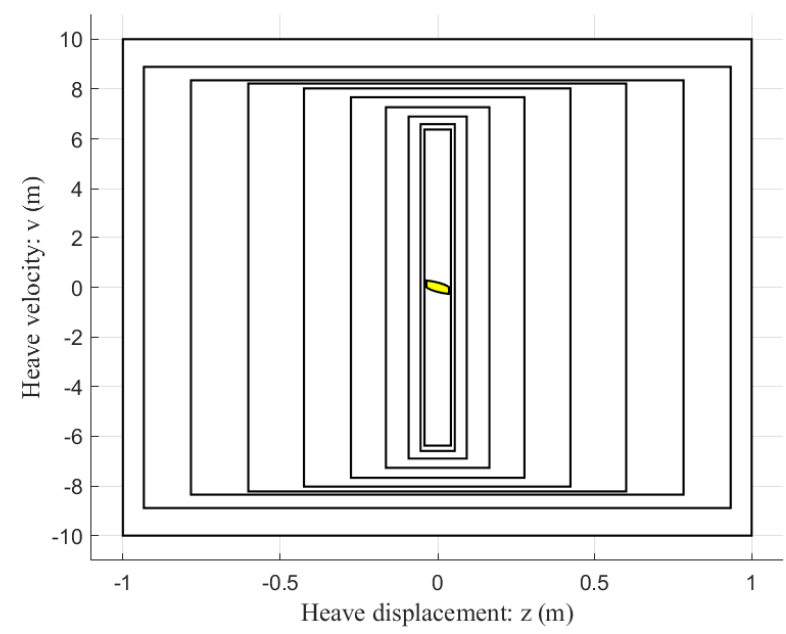

Fig. 4. The projection of tightened state and terminal constraints on $x_{1}-x_{2}$ plane. (the write contours from outside to inside represent tightened state constraints $\mathbb{X}_{0}, \mathbb{X}_{1}, \ldots, \mathbb{X}_{9}$; the yellow contour is the projection of the tightened terminal constraints $\mathbb{X}_{T}$.

assumed to be available at each sampling time using a wave prediction technique. The observer gain $L$ is designed as

$L=\left[\begin{array}{ccccc}0.9412 & -1.1639 & 0.5283 & -20.5234 & -4.4657 \\ 0.0980 & 0.9400 & -3.1529 & 31.9104 & 7.4290\end{array}\right]^{T}$

The estimation error of current wave excitation force is assumed to satisfy $\left|w_{k \mid k}-w_{k}\right| \leq 10 \% w_{\max }$, which with (18), corresponds to the resultant error bound $\mathbb{E}=$ $\left\{e \in \mathbb{R}^{5}:\left|C_{z} e\right| \leq 0.01\right\}$. The feedback gain $F$ is designed offline from (23). In this paper, we adopt a similar approach as [29] which gives

$$
F=\left[\begin{array}{lllll}
-624.92 & -1231.1 & 0.1471 & -0.5744 & 1.1960
\end{array}\right]
$$

and the maximal wave excitation bound for the MOAS $\Sigma$ to exist is $w_{\max }=3.52 \mathrm{kN}$. This means that the economic feedback MPC can safely work in all the sea states whose maximal wave excitation force is no more than $3.52 \mathrm{kN}$.

To facilitate the economic feedback MPC design, we compute the tightened state constraints $\mathbb{X}_{k}$ for $k \in \mathbb{I}_{[0,9]}$, the tightened input constraints $\mathbb{U}_{k}$ for $k \in \mathbb{I}_{[0,9]}$ and the terminal constraints $\mathbb{X}_{T}$ using (21). Fig. 4 shows the projection of the tightened state constraints $\mathbb{X}_{k}$ for $k \in \mathbb{I}_{[0,9]}$ and the tightened terminal constraint $\mathbb{X}_{T}$ on $x_{1}-x_{2}$ plane. Since the system is of the $5^{t h}$ order, it is not possible to directly plot the sets as they are in a 5-dimensional Euclidean space. For better visualization and demonstration purpose, we project the sets

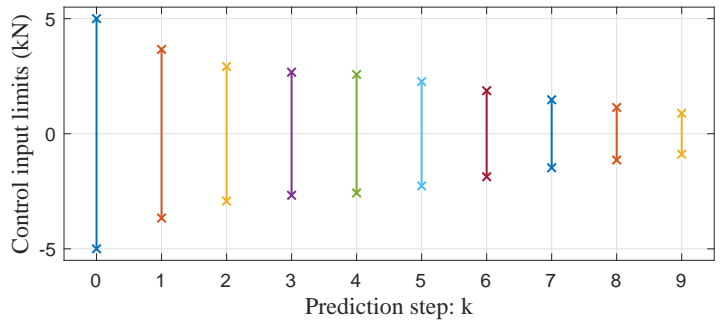

Fig. 5. The tightened control input constraints set $\mathbb{U}_{k}$ for $k \in \mathbb{I}_{[0,9]}$.

from a 5-dimensional Euclidean space into a 2-dimensional Euclidean space where each axis has physical meaning, i.e. the $x_{1}-x_{2}$ plane.

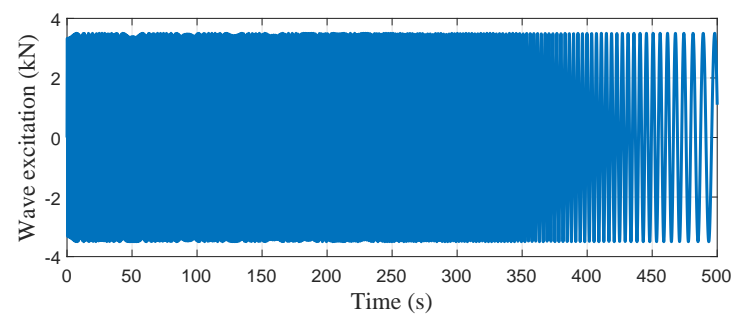

Fig. 6. 500-s of chriping signal used for the time simulation. The magnitude is 3.52 and frequencies cover $0.1-1 \mathrm{~Hz}$
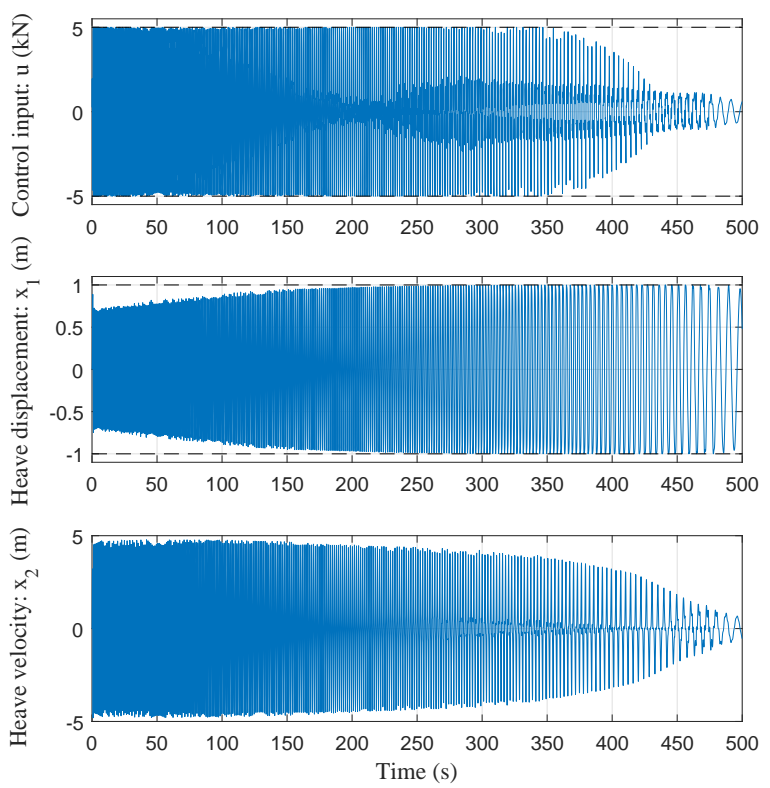

Fig. 7. State and control input responses using the economic feedback MPC proposed in this paper.

The objective function of (24) is employed, where the coefficient $r$ is designed using (27). Since the original cost function with $r=0$ cannot yield a convex optimization with respect to $c$, the minimal $r$ for the resultant objective function to be convex is computed as $r=3.518 \times 10^{-4}$. 


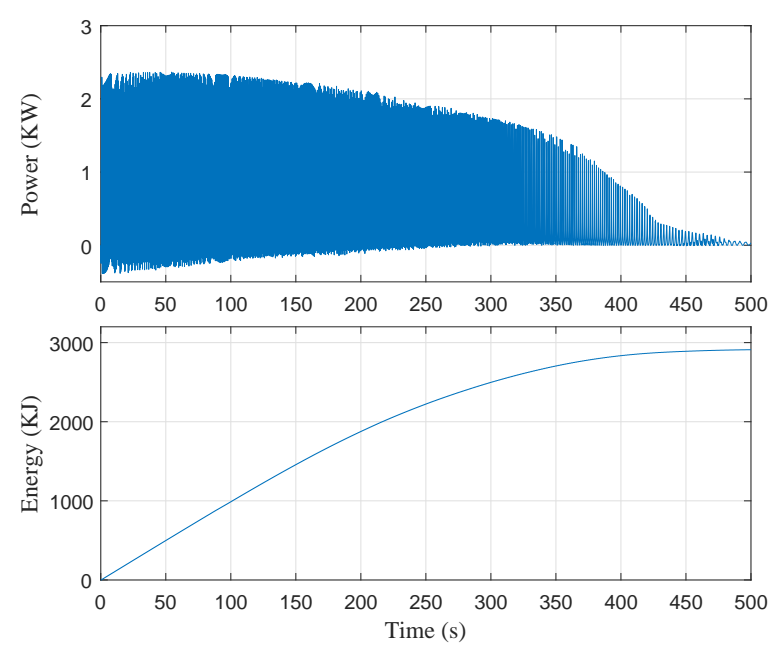

Fig. 8. Power and energy output using the economic feedback MPC proposed in this paper.

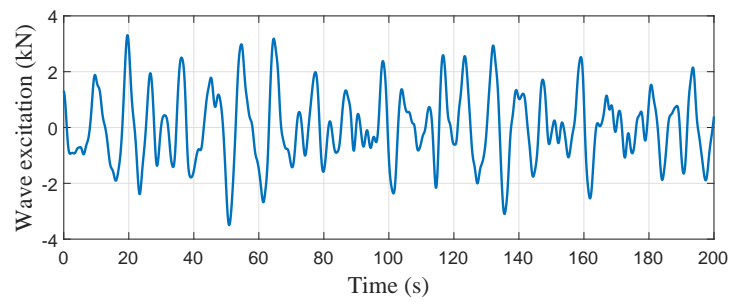

Fig. 9. 200-s of wave excitation used for the time simulation, generated by real sea waves.

To test the constraint satisfaction and robustness of the methods, a segment of 500-s chirp signal shown in Fig. 6 is used as the excitation force. The magnitude of the chirp signal is $3.52 \mathrm{kN}$, and the frequencies sweep from $1 \mathrm{~Hz}$ to $0.1 \mathrm{~Hz}$.

Fig. 7 shows the state and input trajectories. We can see that although the trajectories are generally very close to the boundaries to achieve better performance in terms of the energy output, the state and input constraints are straightly satisfied.

Fig. 8 shows the energy output. We can see that a significant amount of energy is produced during the $0-350 \mathrm{~s}$, corresponding to $1-0.37 \mathrm{~Hz}$. This result shows that besides large operational range, the economic feedback MPC proposed in this paper also has satisfactory power absorption performance.

\section{B. Simulation set 2: comparsive simulation results between the existing MPC and the novel MPC.}

In Subsection V-B, to further test the efficacy of the proposed economic feedback MPC, a set of comparative simulations is provided based on real sea wave data gathered off the coast at Cornwall, UK, which generate the following wave excitation force profile shown in Fig. 9, after scaling according to the size of the point absorber.

For the proposed economic feedback MPC and existing WEC MPC [13], we adopt the same objective function form (24). As calculated in Subsection V-A, the novel MPC is designed with $r=3.518 \times 10^{-4}$. However, constraints violation

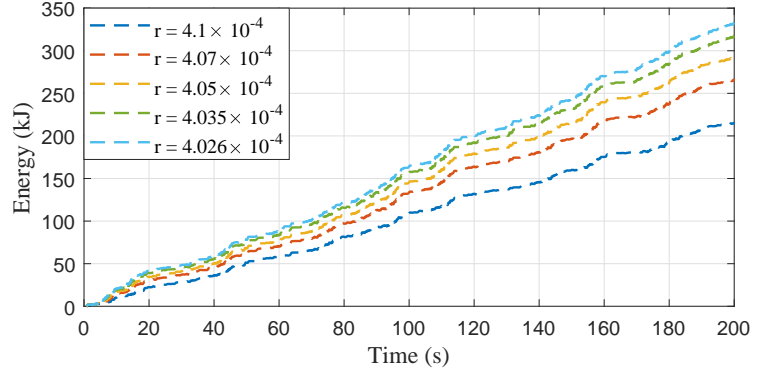

Fig. 10. Energy output comparasion using the existing MPC [13] with different tuning of $r$.
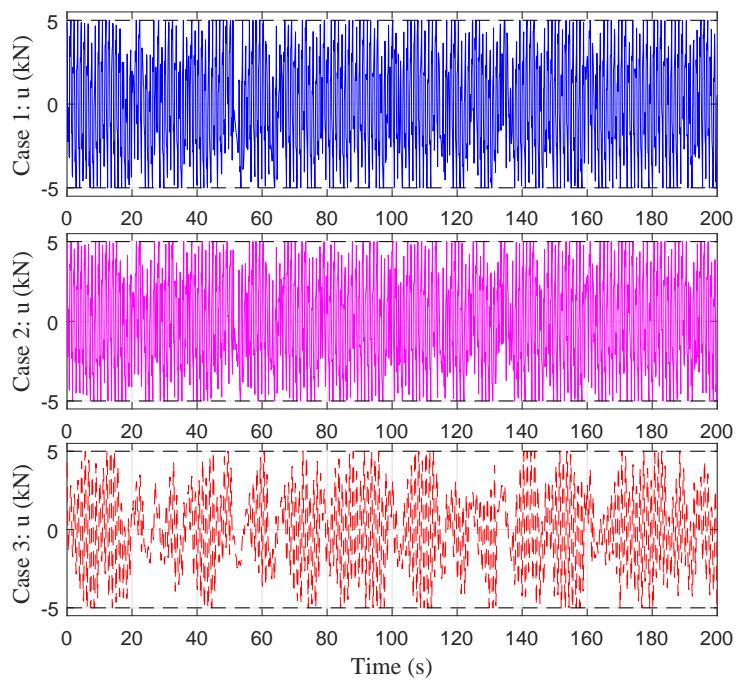

Fig. 11. Control input response comparasion. The proposed economic feedback MPC with accurate predictions (blue dash line); the proposed economic feedback MPC with inaccurate predictions (magenta dot line); the existing MPC [13] (red dash line).

occurs and the existing MPC results in an infeasiblility issue, when using the existing MPC with the same $r=3.518 \times 10^{-4}$.

TABLE II

ENERGY OUTPUT COMPARASION TABLE USING THE EXISTING OPEN-LOOP MPC WITH DIFFERENT TUNING OF $r$.

\begin{tabular}{|c|c|c|c|c|c|c|}
\hline$r\left(\times 10^{-4}\right)$ & 4.1 & 4.07 & 4.05 & 4.035 & 4.026 & 4.025 \\
\hline Energy (kJ) & 215.5 & 266.5 & 293.4 & 317.8 & 333.2 & Infeasible \\
\hline
\end{tabular}

By trial and error, we find that the weight $r$ need to be tuned $r \geq 4.026 \times 10^{-4}$ to guarantee the feasibility and constraints satisfaction. Otherwise, for example, the existing MPC with $r=4.025 \times 10^{-4}$ cannot yield a feasible solution at $44.4 \mathrm{~s}$.

Since the existing MPC can be tuned with different $r$ via trial and error, before moving to the comparisons between different MPC strategies, we first investigate the relationship between the energy output and different turning $r$. Fig. 10 shows the energy output when the WEC is controlled by the existing MPC with different turnings, whose result is summarized in Table II. We can see that the best energy output tuning scenario is $333.2 \mathrm{~kJ}$ with $r=4.026 \times 10^{-4}$.

Figs. 11-14 shows the comparative time simulations among 


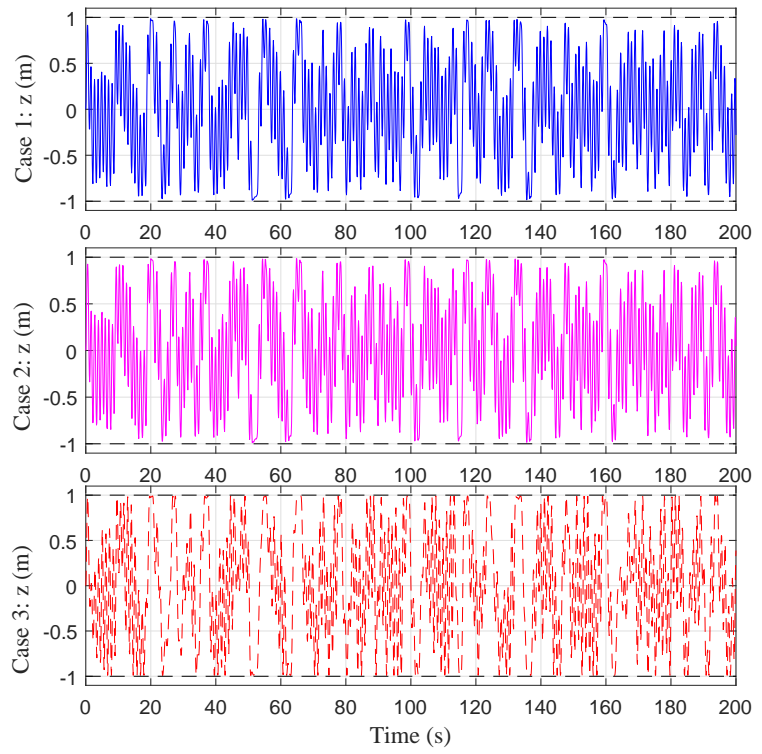

Fig. 12. Heave displacement response comparasion. The proposed economic feedback MPC with accurate predictions (blue dash line); the proposed economic feedback MPC with inaccurate predictions (magenta dot line); the existing MPC [13] (red dash line).
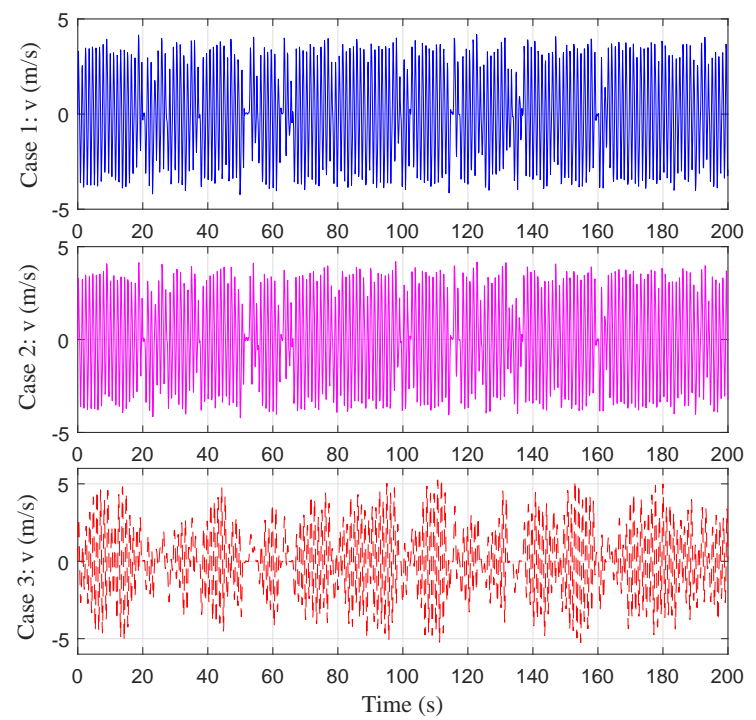

Fig. 13. Heave velocity response comparasion. The proposed economic feedback MPC with accurate predictions (blue dash line); the proposed economic feedback MPC with inaccurate predictions (magenta dot line); the existing MPC [13] (red dash line).

- Case 1: the proposed novel economic feedback MPC with accurate wave excitation predictions (blue solid line);

- Case 2: the proposed novel economic feedback MPC with predictions subject to $10 \%$ of prediction error (magenta solid line);

- Case 3: the existing open-loop MPC [13] with the best tuning via trial and error $r=4.026 \times 10^{-4}$ (red dash line).

Note that in this case, the existing MPC is tuned for maximal energy output only purely based on trial and error,

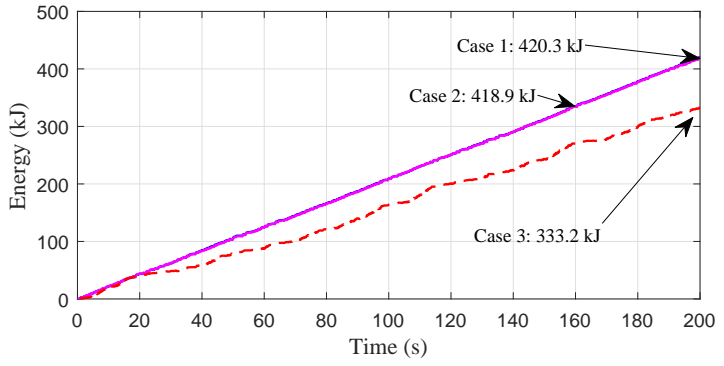

Fig. 14. Energy output comparasion. The proposed economic feedback MPC with accurate predictions (blue dash line); the proposed economic feedback MPC with inaccurate predictions (magenta dot line); the existing open-loop MPC [13] (red dash line).

which in fact, can easily lose its efficacy in similar sea scenarios.

Here in the Cases 1 and 3, wave excitation force prediction is assumed to be accurate. However, the predictions obtained from start-of-the-art prediction techniques inevitably come with uncertainties. To further study the influence of the prediction errors in the performance of the proposed control method, we deliberately add $10 \%$ of statistical prediction discrepancies in case 2 , which is notably greater than those with the stateof-the-art wave prediction techniques [31], [32].

Figs. 11-13 shows the control input responses, heave displacement response and heave velocity response, respective. The state and control input constraints are satisfied for the three cases. We can also find that the maximal magnitudes of the state and control input are very similar, which not only shows a fair comparison basis, but also demonstrates that both control methods are pushed to their maximum limits for energy conversion.

Fig. 14 compares the energy outputs of the three cases. The energy output using the existing MPC and the economic feedback MPC are $333.2 \mathrm{~kJ}$ and $420.3 \mathrm{~kJ}$ respectively, which represents an $26.1 \%$ of energy increase. This is partially due to the fact that the state and input trajectories shown in Figs. 11-12 using the economic feedback MPC are closer to the boundary most of the time compared with the existing MPC, which means the limits of the WEC design and the PTO mechanism (e.g. the torque of a PTO generator) are utilized much more efficiently. Fig. 14 also shows that by using the inaccurate predictions, the energy output is $418.9 \mathrm{~kJ}$, which represents a very minor performance degradation compared with using the accurate predictions. The result clearly shows that the proposed economic feedback MPC is robust to wave excitation forecasting inaccuracies.

\section{Simulation set 3: simulation results based on JONSWAP spectrum.}

In this subsection, simulations based on different JONSWAP spectrum are presented to show the efficacy of the proposed economic feedback MPC method in different sea scenarios. Fig. 15 demonstrates the energy output based on JONSWAP spectrum with a significant wave height $H_{s}=1.2 \mathrm{~m}$ and a peak period $T_{p}=4 \mathrm{~s}$. In this case, the existing MPC need to be retuned via trial and error to retain the maximal possible 


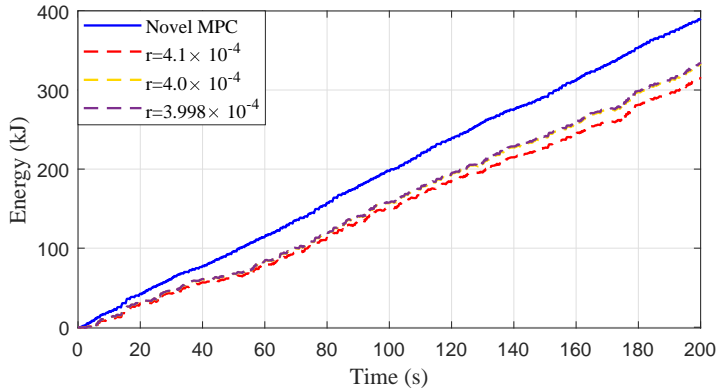

Fig. 15. Energy output comparasion. The proposed economic feedback MPC with accurate predictions (blue solid line); The existing MPC [13] with different tuning: $r=4.1 \times 10^{-4}$ (red dash line); $r=4.0 \times 10^{-4}$ (yellow dash line); $r=3.998 \times 10^{-4}$ (purple dash line).

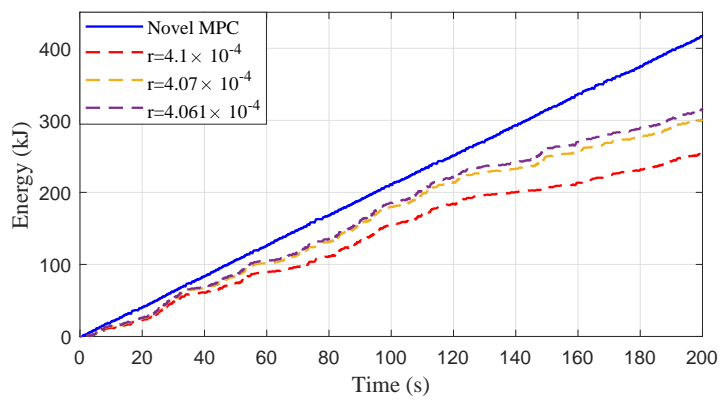

Fig. 16. Energy output comparasion. The novel MPC with accurate predictions (blue solid line); The existing MPC with different tuning: $r=4.1 \times$ $10^{-4}$ (red dash line); $r=4.07 \times 10^{-4}$ (yellow dash line); $r=4.061 \times 10^{-4}$ (purple dash line).

energy output, whilst the novel MPC proposed in this paper uses the same tuning. This clearly shows the advantage of the novel MPC framework for its simple design. The energy output of the novel MPC is $390.8 \mathrm{~kJ}$ while the energy outputs of the existing MPC with $r=4.1 \times 10^{-4}, r=4.0 \times 10^{-4}$ and $3.998 \times 10^{-4}$ are $315.1 \mathrm{~kJ}, 332.3 \mathrm{~kJ}$ and $333.8 \mathrm{~kJ}$, respectively. Note that if we further tune the existing MPC with $r=3.997 \times$ $10^{-4}$, the infeasibility and constraints violation occur.

Fig. 16 shows the energy output based on JONSWAP spectrum with significant wave height $H_{s}=1.2 \mathrm{~m}$ and peak period $T_{p}=7 \mathrm{~s}$. In this case, the existing MPC with neither $r=3.998 \times 10^{-4}$ nor $r=4.026 \times 10^{-4}$ as tuned in Subsection V-B can ensure feasibility for the $200 \mathrm{~s}$. To avoid constraints violation and infeasibility, the weights $r$ need to be retuned to $r \geq 4.061 \times 10^{-4}$. The energy output of the novel MPC is $417.3 \mathrm{~kJ}$ while the energy output of the existing MPC with the best possible tuning $r \geq 4.061 \times 10^{-4}$ is $312.1 \mathrm{~kJ}$, which represents an $33.7 \%$ of energy output improvement.

The average time of running a step of the simulation using the novel economic feedback MPC and the existing MPC are $0.0116 \mathrm{~s}$ and $0.0067 \mathrm{~s}$, respectively. This result indicates the additional computational burden of using economic feedback MPC compared with using existing MPC is minor and both of them are significantly smaller than the sampling time $t_{s}=0.1$ s.

\section{CONCLUSION}

In this paper, we proposed an economic feedback model predictive control (MPC) scheme that not only can prominently improve energy conversion efficiency of wave energy converters (WECs), but also guarantee their safe operation over a wide range of sea conditions. Safety features as the recursive feasibility and robust constraints satisfaction are guaranteed, which can remarkably boost its application potential. The effect required to tune the controller is also notably reduced.

This paper also provides a sufficient condition guaranteeing the safe operation of WECs, which can be useful for WEC operation engineers to switch off the WEC to avoid device damage when the incoming wave excitation magnitude exceeds the maximum designed bound $w_{\max }$. This condition can also be used as a guideline for WEC design engineers to modify their designs of WECs and PTO mechanisms so that the modified WECs can operate in a wide range of sea states.

\section{APPENDIX}

\section{NOMENCLATURE}

\begin{tabular}{ll}
\hline Abbreviations & Explanation \\
\hline MPC & Model Predictive Control \\
WEC & Wave Energy Converter \\
EM & Energy Maximization \\
PTO & Power Take-off \\
PA & Point Absorber \\
QP & Quadratic Programming \\
MOAS & Maximal Output Admissible Set \\
JONSWAP & Joint North Sea Wave Project \\
\hline
\end{tabular}

The FORMULATION OF $\mathcal{H}, \mathcal{F}, \mathcal{G}$ IN (26)

From (25), we have the predicted heave velocity and control input trajectories $\tilde{\boldsymbol{v}}_{k}:=\left[C_{v} \tilde{x}_{k \mid k}, \ldots, C_{v} \tilde{x}_{k+n_{p}-1 \mid k}\right]$ and $\tilde{\boldsymbol{u}}_{k}:=\left[\tilde{u}_{k \mid k}, \tilde{u}_{k+1 \mid k}, \ldots, \tilde{u}_{k+n_{p}-1 \mid k}\right]$ can be calculated by

$$
\begin{aligned}
\tilde{\boldsymbol{v}}_{k} & =\mathcal{M}_{v} \hat{x}_{k}+\mathcal{C}_{c v} \boldsymbol{c}_{k}+\mathcal{C}_{w v} \boldsymbol{w}_{k} \\
\tilde{\boldsymbol{u}}_{k} & =\mathcal{M}_{u} \hat{x}_{k}+\mathcal{C}_{c u} \boldsymbol{c}_{k}+\mathcal{C}_{w u} \boldsymbol{w}_{k}
\end{aligned}
$$

where

$$
\begin{aligned}
& \mathcal{M}_{v}:=\left[\begin{array}{c}
C_{v} \\
C_{v} A_{F} \\
\vdots \\
C_{v} A_{F}^{n_{p}-1}
\end{array}\right], \quad \mathcal{M}_{u}:=\left[\begin{array}{c}
I \\
F A_{F} \\
\vdots \\
F A_{F}^{n_{p}-1}
\end{array}\right] \\
& \mathcal{C}_{c v}:=\left[\begin{array}{ccccc}
0 & & & & \\
C_{v} B_{u} & 0 & & & \\
C_{v} A_{F} B_{u} & C_{v} B_{u} & 0 & & \\
\vdots & \ddots & \ddots & \ddots & \\
C_{v} A_{F}^{n_{p}-2} B_{u} & \ldots & C_{v} A_{F} B_{u} & C_{v} B_{u} & 0
\end{array}\right] \\
& \mathcal{C}_{w v}:=\left[\begin{array}{ccccc}
0 & & & & \\
B_{w} & 0 & & & \\
A_{F} B_{w} & B_{w} & 0 & & \\
\vdots & \ddots & \ddots & \ddots & \\
A_{F}^{n_{p}-2} B_{w} & \ldots & A_{F} B_{w} & B_{w} & 0
\end{array}\right]
\end{aligned}
$$




$$
\begin{aligned}
\mathcal{C}_{c u}:= & {\left[\begin{array}{ccccc}
1 & 0 & & & \\
B_{u} & 1 & 0 & & \\
A_{F} B_{u} & B_{u} & 1 & 0 & \\
\vdots & \ddots & \ddots & \ddots & \ddots \\
A_{F}^{n_{p}-2} B_{u} & \ldots & A_{F} B_{u} & B_{u} & 1
\end{array}\right] } \\
\mathcal{C}_{w u}:= & {\left[\begin{array}{ccccc}
0 & & & & \\
F B_{w} & 0 & & & \\
F A_{F} B_{w} & B_{w} & 0 & & \\
\vdots & \ddots & \ddots & \ddots & \\
F A_{F}^{n_{p}-2} B_{w} & \ldots & A_{F} B_{w} & F B_{w} & 0
\end{array}\right] }
\end{aligned}
$$

with $A_{F}:=A+B_{u} F$ and (24) can be written in matrix multiplications $r \tilde{\boldsymbol{u}}_{k}^{T} \tilde{\boldsymbol{u}}_{k}+\tilde{\boldsymbol{u}}_{k}^{T} \tilde{\boldsymbol{v}}_{k}$. With (35), we have that the $\mathcal{H}, \mathcal{F}, \mathcal{G}$ in (26) can be determined by

$$
\begin{aligned}
\mathcal{H}= & r \mathcal{C}_{c u}^{T} \mathcal{C}_{c u}+(1 / 2)\left(\mathcal{C}_{c u}^{T} \mathcal{C}_{c x}+\mathcal{C}_{c x}^{T} \mathcal{C}_{c u}\right) \\
\mathcal{F}= & \left(\mathcal{C}_{c v}+r \mathcal{C}_{c u}\right)^{T}\left(\mathcal{M}_{u} \hat{x}_{k}+\mathcal{C}_{w u} \boldsymbol{w}_{k}\right) \\
& +\mathcal{C}_{c u}^{T}\left(\mathcal{M}_{v} \hat{x}_{k}+\mathcal{C}_{w v} \boldsymbol{w}_{k}\right) \\
\mathcal{G}= & \left(\mathcal{M}_{u} \hat{x}_{k}+\mathcal{C}_{w u} \boldsymbol{w}_{k}\right)^{T}\left(\mathcal{M}_{v} \hat{x}_{k}+\mathcal{C}_{w v} \boldsymbol{w}_{k}\right) \\
& +r\left(\mathcal{M}_{v} \hat{x}_{k}+\mathcal{C}_{w v} \boldsymbol{w}_{k}\right)^{T}\left(\mathcal{M}_{v} \hat{x}_{k}+\mathcal{C}_{w v} \boldsymbol{w}_{k}\right)
\end{aligned}
$$

\section{REFERENCES}

[1] K. Gunn and C. Stock-Williams, "Quantifying the global wave power resource," Renewable Energy, vol. 44, pp. 296-304, 2012.

[2] A. Clément, P. McCullen, A. Falcão, A. Fiorentino, F. Gardner, K. Hammarlund, G. Lemonis, T. Lewis, K. Nielsen, S. Petroncini et al., "Wave energy in europe: current status and perspectives," Renewable and sustainable energy reviews, vol. 6, no. 5, pp. 405-431, 2002.

[3] B. Drew, A. Plummer, and M. N. Sahinkaya, "A review of wave energy converter technology," Proceedings of the Institution of Mechanical Engineers, Part A: Journal of Power and Energy, vol. 223, no. 8, pp. 887-902, 2009.

[4] J. Ringwood, G. Bacelli, and F. Fusco, "Energy-maximizing control of wave-energy converters: The development of control system technology to optimize their operation," Control Systems, IEEE, vol. 34, no. 5, pp. 30-55, 2014.

[5] N. Faedo, G. Scarciotti, A. Astolfi, and J. V. Ringwood, "Energymaximising control of wave energy converters using a moment-domain representation," Control Engineering Practice, vol. 81, pp. 85-96, 2018.

[6] J. Falnes, Ocean Waves and Oscillating Systems. Cambridge University Press, 2002.

[7] M. Ellis, H. Durand, and P. D. Christofides, "A tutorial review of economic model predictive control methods," Journal of Process Control, vol. 24 , no. 8, pp. 1156-1178, 2014.

[8] J. B. Rawlings and R. Amrit, "Optimizing process economic performance using model predictive control," in Nonlinear model predictive control. Springer, 2009, pp. 119-138.

[9] M. Diehl, R. Amrit, and J. B. Rawlings, "A lyapunov function for economic optimizing model predictive control," IEEE Transactions on Automatic Control, vol. 56, no. 3, pp. 703-707, 2011.

[10] M. Muller and L. Grune, "Economic model predictive control without terminal constraints for optimal periodic behavior," Automatica, vol. 70, pp. 128-139, 2016.

[11] D. Angeli, R. Amrit, and J. B. Rawlings, "On average performance and stability of economic model predictive control," IEEE transactions on automatic control, vol. 57, no. 7, pp. 1615-1626, 2012.

[12] S. Zhan, J. Na, G. Li, and B. Wang, "Adaptive model predictive control of wave energy converters," IEEE Transactions on Sustainable Energy (Available Online), pp. 1-1, 2018.

[13] G. Li and M. R. Belmont, "Model predictive control of sea wave energy converters-part i: A convex approach for the case of a single device," Renewable Energy, vol. 69, pp. 453-463, 2014.

[14] L. Chisci, J. A. Rossiter, and G. Zappa, "Systems with persistent disturbances: predictive control with restricted constraints," Automatica, vol. 37, no. 7, pp. 1019-1028, 2001.
[15] D. Q. Mayne, J. B. Rawlings, C. V. Rao, and P. O. Scokaert, "Constrained model predictive control: Stability and optimality," Automatica, vol. 36 , no. 6, pp. 789-814, 2000.

[16] P. J. Goulart, E. C. Kerrigan, and J. M. Maciejowski, "Optimization over state feedback policies for robust control with constraints," Automatica, vol. 42, no. 4, pp. 523-533, 2006.

[17] M. Trapanese, V. Boscaino, G. Cipriani, D. Curto, V. Di Dio, and V. Franzitta, "A permanent magnet linear generator for the enhancement of the reliability of a wave energy conversion system," IEEE Transactions on Industrial Electronics (Available Online), 2018.

[18] O. Farrok, M. R. Islam, M. R. I. Sheikh, Y. Guo, and J. G. Zhu, "A split translator secondary stator permanent magnet linear generator for oceanic wave energy conversion," IEEE Transactions on Industrial Electronics (Available Online), 2018.

[19] P. Ricci, J. Lopez, M. Santos, P. Ruiz-Minguela, J. Villate, F. Salcedo et al., "Control strategies for a wave energy converter connected to a hydraulic power take-off," IET renewable power generation, vol. 5, no. 3, pp. 234-244, 2011

[20] M. Mueller, "Electrical generators for direct drive wave energy converters," IEE Proceedings-generation, transmission and distribution, vol. 149, no. 4, pp. 446-456, 2002.

[21] P. Stansby, E. C. Moreno, and T. Stallard, "Large capacity multi-float configurations for the wave energy converter $\mathrm{m} 4$ using a time-domain linear diffraction model," Applied Ocean Research, vol. 68, pp. 53-64, 2017.

[22] Z. Yu and J. Falnes, "State-space modelling of a vertical cylinder in heave," Applied Ocean Research, vol. 17, no. 5, pp. 265-275, 1995.

[23] S. Zhan and G. Li, "Linear noncausal optimal control of wave energy converters," IEEE Transactions on Control System Technology (Available Online), 2018

[24] M. Penalba, T. Kelly, and J. V. Ringwood, "Using nemoh for modelling wave energy converters: A comparative study with wamit," in Proceedings of the 12th European Wave and Tidal Energy Conference (EWTEC2017), Cork, Ireland, 2017, p. 631.

[25] D. Q. Mayne, S. Raković, R. Findeisen, and F. Allgöwer, "Robust output feedback model predictive control of constrained linear systems," Automatica, vol. 42, no. 7, pp. 1217-1222, 2006.

[26] I. Kolmanovsky and E. G. Gilbert, "Maximal output admissible sets for discrete-time systems with disturbance inputs," in American Control Conference, Proceedings of the 1995, vol. 3. IEEE, 1995.

[27] E. C. Kerrigan, "Robust constraint satisfaction: Invariant sets and predictive control," Ph.D. dissertation, University of Cambridge, 2001.

[28] S. V. Rakovic, E. C. Kerrigan, K. I. Kouramas, and D. Q. Mayne, "Invariant approximations of the minimal robust positively invariant set," IEEE Transactions on Automatic Control, vol. 50, no. 3, pp. 406-410, 2005.

[29] J. Löfberg, Minimax approaches to robust model predictive control. Linköping University Electronic Press, 2003, vol. 812.

[30] K. Hasselmann, T. Barnett, E. Bouws, H. Carlson, D. Cartwright, K. Enke, J. Ewing, H. Gienapp, D. Hasselmann, P. Kruseman et al., "Measurements of wind-wave growth and swell decay during the joint north sea wave project (jonswap)," Ergänzungsheft 8-12, 1973.

[31] M. Belmont, J. Christmas, J. Dannenberg, T. Hilmer, J. Duncan, J. Duncan, and B. Ferrier, "An examination of the feasibility of linear deterministic sea wave prediction in multidirectional seas using wave profiling radar: Theory, simulation, and sea trials," Journal of Atmospheric and Oceanic Technology, vol. 31, no. 7, pp. 1601-1614, 2014.

[32] A. Mérigaud and J. V. Ringwood, "Incorporating ocean wave spectrum information in short-term free-surface elevation forecasting," IEEE Journal of Oceanic Engineering, 2018.

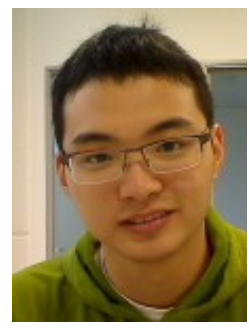

Siyuan Zhan received the bachelor's degree from Shanghai Jiao Tong University, Shanghai, China, in 2013, the master's degree from the University of Pennsylvania, Philadelphia, PA, USA, in 2014 and $\mathrm{Ph} . \mathrm{D}$. degree from the Queen Mary University of London, London, U.K., in 2018, respectively. He is currently a Postdoctoral Research Assistant with the Queen Mary University of London, London, U.K. His current research interests include constrained optimal control, adaptive control, model predictive control, and control applications in renewable energy 


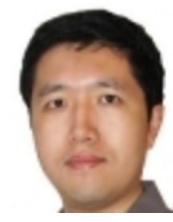

Guang Li (M'09) received his Ph.D. degree in Electrical and Electronics Engineering, specialized in control systems, from the University of Manchester, in 2007. He is currently a Senior Lecturer in dynamics modeling and control in Queen Mary University of London, UK. His current research interests include constrained optimal control, model predictive control, adaptive robust control and control applications including renewable energies and energy storage, etc.

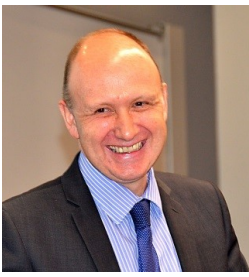

Colin Bailey was born in Hillingdon, UK on 11/04/67. He received a Doctorate in Civil and Structural Engineering (1995) and a First Class Honours (BEng) in Civil and Structural Engineering (1992), from the University of Sheffield; a Higher National Certificate (HNC) in Civil Engineering (1988) from Richmond College and a National Certificate (ONC) in Civil Engineering (1986) from Slough College.

Professor Bailey is a Fellow of The Royal Academy of Engineering (FREng), a Fellow of The Institution of Civil Engineers (FICE), a Fellow of The Institution of Structural Engineers (FIStructE) and a Member of The Institution of Fire Engineers (MIFireE). He has published over 130 papers and design guides/books and has been presented with 9 prizes for his research work. He is currently President and Principal of Queen Mary University of London (from 2017). He was previously Deputy President and Deputy Vice-Chancellor(2014-2017), Vice-President and Dean of the Faculty of Engineering and Physical Sciences (2009-2014), Head of School of Mechanical, Aerospace and Civil Engineering (2007-2009), Head of Dynamics, Structures \& Design Group (2004-2007) and Head of Structural Engineering Division, UMIST (2002-2004), at the University of Manchester. 